\title{
The Inclination Angle and Mass of the Black Hole in XTE J1118+480
}

\author{
Dawn M. Gelino \\ Michelson Science Center, California Institute of Technology, $770 \mathrm{~S}$ Wilson Ave. \\ Pasadena, CA 91125 \\ Solen Balman, Umit Kiziloglu, and Arda Yilmaz \\ Middle East Technical University, Ankara, Turkey \\ Emrah Kalemci \\ Sabanci University, Orhanli-Tuzla, Istanbul, Turkey \\ John A. Tomsick \\ Space Sciences Laboratory, University of California, Berkeley, CA 94720
}

\begin{abstract}
We have obtained optical and infrared photometry of the quiescent soft X-ray transient XTE J1118+480. In addition to optical and $J$-band variations, we present $H$ - and $K_{s}$-band ellipsoidal variations for this system. We model the variations in all bands simultaneously with the WD98 light curve modeling code. The infrared colors of the secondary star in this system are consistent with those of a $\mathrm{K} 7 \mathrm{~V}$, while there is evidence for light from the accretion disk in the optical. Combining the models with the observed spectral energy distribution of the system, the most likely value for the orbital inclination angle is $68^{\circ} \pm 2^{\circ}$. This inclination angle corresponds to a primary black hole mass of $8.53 \pm 0.60 M_{\odot}$. Based on the derived physical parameters and infrared colors of the system, we determine a distance of $1.72 \pm 0.10 \mathrm{kpc}$ to XTE J1118+480.
\end{abstract}

\section{Introduction}

XTE J1118+480 is a high Galactic latitude $\left(b=+62^{\circ}\right)$ system that has been observed by numerous groups over many wavelength regimes. Recent orbital parameters determined from optical spectra suggest an orbital period of $4.078 \mathrm{hr}$ and a secondary star radial velocity semi-amplitude of $709 \pm 7 \mathrm{~km} \mathrm{~s}^{-1}$ (Torres et al. 2004). These values imply a mass function of $f(M)=6.3 \pm 0.2 \mathrm{M}_{\odot}$, identifying the compact object as a black hole.

Determining a precise black hole mass requires an accurate measurement of the orbital inclination angle, $i$, of the system. Since the amplitude of the secondary star ellipsoidal variations (which result from the tidal and rotational distortions of the Roche lobe-filling star, as well as its non-uniform surface brightness distribution) is a strong function of $i$, we can model these variations to constrain $i$. Simultaneously modeling light curves that span more than one wavelength regime provides tighter constraints than modeling IR light curves alone. Previous inclination estimates for XTE J1118+480 have come from modeling optical ellipsoidal variations as the system approached quiescence (McClintock et al. 2001; Wagner et al. 2001; Zurita et al. 2002). These inclination angles range from $55^{\circ}$ (McClintock et al. 2001) to $83^{\circ}$ (Wagner et al. 2001), and correspond to primary masses of $10 \mathrm{M}_{\odot}$ and 6.0 $\mathrm{M}_{\odot}$, respectively. Since this system has been known to exhibit optical superhumps from 

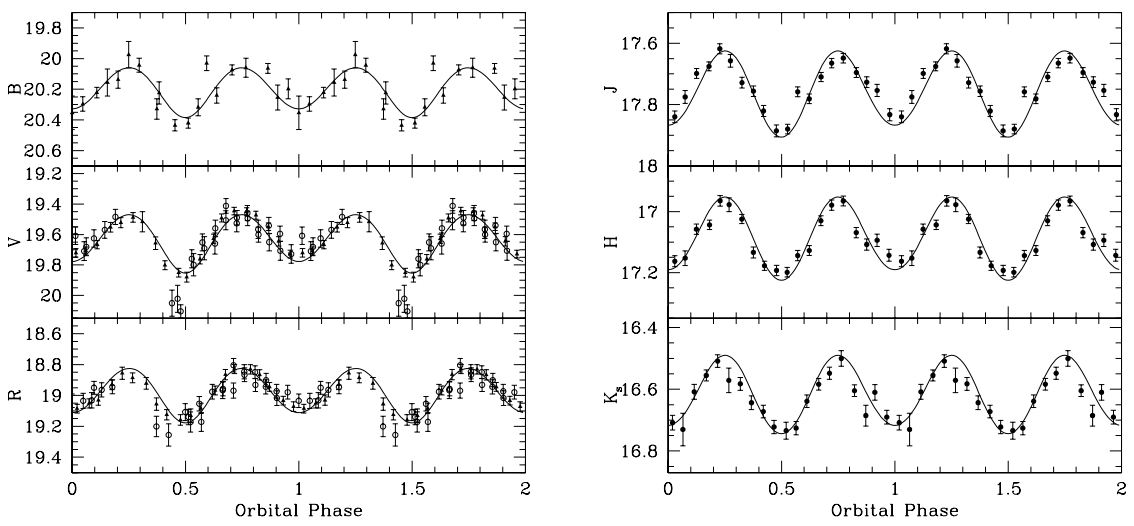

Figure 1. Left: XTE J1118+480 B-, $V$-, and $R$-band light curves from 2003 (open circles) and 2004 (filled triangles). Right: $J_{-}, H$-, and $K_{s}$-band light curves (circles). All data are plotted over two phase cycles for clarity. Error bars are $1 \sigma$. The solid line represents the best fitting $\left(i=68^{\circ}\right)$ WD98 model.

the precession of an eccentric accretion disk on its way to quiescence (Zurita et al. 2002), it is important to determine the orbital inclination angle while XTE J1118+480 is in a truly quiescent state. In order to determine an accurate orbital inclination angle for XTE J1118+480, we obtained $B-, V-, R-, J_{-}, H_{-}$, and $K_{s}$-band light curves of the system while in quiescence, and simultaneously modeled them with the WD98 light curve modeling code (Wilson 1998). The modeled inclination angle was then combined with published orbital parameters to determine a highly constrained mass of the black hole in this X-ray binary.

\section{Data Reduction and Modeling}

Details of the data acquisition, reduction, modeling and analysis can be found in Gelino et al. (2006). The optical data were obtained with the CCD on the $1.5 \mathrm{~m}$ telescope at the TUBITAK National Observatory in Turkey over 7 nights in 2003 and 2004. The IR data were obtained with SQIID on the $2.1 \mathrm{~m}$ telescope at the Kitt Peak National Observatory over 2 nights in 2003. All reduced data were simultaneously modeled with the WD98 light curve modeling code as described in Gelino et al. (2006). The resulting light curves and best fit models are shown in Figure 1. We used a spectral energy distribution (SED; Fig 2) to determine an estimate for the amount of diluting (i.e. disk) light in the system. The observed data were compared with SEDs for K0V - M4V stars with $A_{V}=0.045-0.085$ mag. The best-fit SED, normalized at $H$, was that of a K7V (consistent with published results from optical spectra) with $65 \%$ extra light at B and $31 \%$ extra light at V.

\section{Results}

The derived parameters in Table 1 are based on the simultaneous modeling of quiescent ellipsoidal variations including disk contributions of $62 \%$ in the $B$-band and $31 \%$ in the $V$ - 


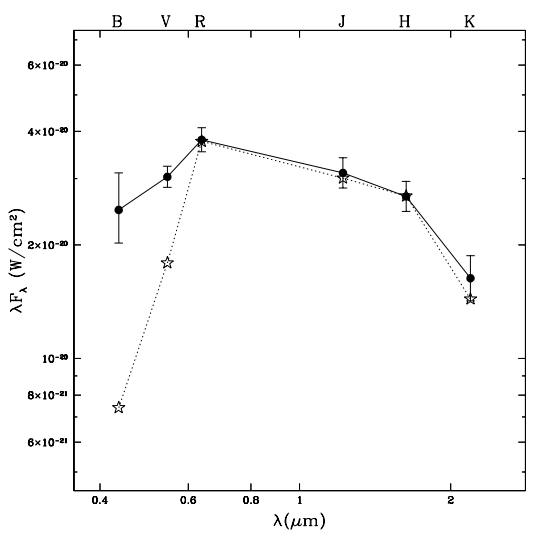

Table 1. Derived Parameters for XTE J1118+480

\begin{tabular}{lc} 
Parameter & Value $^{a}$ \\
\hline Amount of Disk Light at B $(\%)$ & $62 \pm 3$ \\
Amount of Disk Light at V $(\%)$ & $31 \pm 3$ \\
Orbital Inclination Angle $\left({ }^{\circ}\right)$ & $68 \pm 2$ \\
Primary Object Mass $\mathrm{M}_{1}\left(\mathrm{M}_{\odot}\right)$ & $8.53 \pm 0.60$ \\
Secondary Star Mass $\mathrm{M}_{2}\left(\mathrm{M}_{\odot}\right)$ & $0.37 \pm 0.03$ \\
Orbital Separation a $\left(\mathrm{R}_{\odot}\right)$ & $2.67 \pm 0.06$ \\
Secondary Star Radius RL2 $\left(\mathrm{R}_{\odot}\right)$ & $0.43 \pm 0.01$ \\
Distance $(\mathrm{kpc})$ & $1.72 \pm 0.10$ \\
\hline${ }^{a}$ Errors are $1 \sigma\left(\Delta \chi^{2}=1\right)$ &
\end{tabular}

Figure 2. XTE J1118+480 phase-averaged optical-infrared quiescent SED dereddened by $A_{V}=0.065 \mathrm{mag}$ (filled circles). Error bars are $1 \sigma$. The observed data were compared with SEDs for K0V - M4V stars with $\mathrm{A}_{V}=0.045-0.085$ mag. The best fit SED, normalized at $H$, is that of a $\mathrm{K} 7 \mathrm{~V}$ with $65 \%$ extra light at $B$ and $31 \%$ extra light at $V$ (open stars).

band. Consistent with Shahbaz et al. (2005) and Fitzgerald \& Orosz (2003), we do not see evidence for any optical superhump light or irradiation in the system. This supports the the fact that the system was in a truly quiescent state during our observations.

While the orbital inclination angle found here is lower than that found by groups who optically studied the system while approaching quiescence and exhibiting superhumps (Zurita et al. $2002, i=71-82^{\circ}$ ), it is consistent with that found from data taken in true quiescence that suggest no significant superhump activity (Fitzgerald \& Orosz 2003, $i=63-73^{\circ}$ ). Furthermore, the distance we find is consistent with those found previously through both optical (Wagner et al. 2001; McClintock et al. 2001, 1.9 $\pm 0.4 \mathrm{kpc}, 1.8 \pm 0.6 \mathrm{kpc}$, respectively) and infrared (Mikolajewska et al. 2005, $1.4 \pm 0.2 \mathrm{kpc}$ ) observations.

Simultaneously modeling multi-wavelength light curves allows us to better constrain the amount of disk light in an X-ray binary system. As a result, we have been able to constrain the mass of the black hole in the XTE $1118+480$ system to $8.53 \pm 0.60 \mathrm{M}_{\odot}$, and the distance to the system of $1.72 \pm 0.10 \mathrm{kpc}$.

\section{References}

Fitzgerald, M. \& Orosz, J.A., 2003, AAS, \#6.01

Gelino, D. M., et al., 2006, ApJ, 642, 438

McClintock, J., et al., 2001, ApJ, 551, L147

Mikolajewska, J., et al., 2005, MNRAS, 362, L13

Shahbaz, T., et al., 2005, MNRAS, 362, 975

Torres, M., et al., 2004, ApJ, 612, 1026

Wagner, R., et al., 2001, ApJ, 556, 42

Wilson, R.E. 1998, in Reference Manual to the Wilson-Devinney Program, Computing Binary Star Observables, Version 1998 (Gainesville, FL: Univ. Florida)

Zurita, C., et al., 2002, MNRAS, 333, 791 